\section{El programa Autoproducción de \\ Alimentos. Un espacio de intersección entre extensión, investigación y docencia}

\author{
Jimena Verón \\ Docente investigadora de la Facultad \\ de Ciencias Agrarias y de la Facultad \\ de Ciencias de la Salud y Servicio \\ Social. Universidad Nacional de Mar \\ del Plata, Argentina. \\ veronjimena@hotmail.com
}

\section{Virginia Hamdan}

Docente investigadora de la Facultad de Ciencias Agrarias, Universidad Nacional de Mar del Plata. vhamdan@telefax.com.ar Nacional de Mar del Plata.
Integración de la docencia y la extensión /

Intervenciones

RECEPCIÓN: 24/06/16

Paula Natinzon de Ciencias Agrarias, Universidad Investigadora del Instituto Nacional de Tecnología Agropecuaria, Argentina. natinzon.paula@inta.gob.ar

\section{ACEPTACIÓN FINAL: 10/10/16}

\section{Resumen}

El presente trabajo pretende realizar una contribución teórico-metodológica a las experiencias de extensión a partir de la relación que se construye entre investigación, la docencia y la extensión en una práctica concreta: Programa Autoproducción de Alimentos de la Facultad de Ciencias Agrarias de la Universidad Nacional de Mar del Plata y del Instituto Nacional de Tecnología Agropecuaria-Balcarce.

Esta actividad constituye en sí una evidencia de la parcialidad que conlleva la fragmentación entre la extensión, la docencia y la investigación. La dinámica de este tipo de acciones en y con la comunidad genera que tanto estudiantes, profesionales, docentes-investigadores y productores se asuman indistintamente en roles relevantes en cuanto a gestión, extensión, investigación y docencia, en una forma no tradicional pero necesaria para la concreción y sustentabilidad de los proyectos consensuados, planteando interrogantes que hacen a la formación praxiológica no solo de una disciplina sino de la ciencia de manera integral como proceso histórico "que está siendo".

Palabras-clave

- Extensión

- Investigación

- Docencia

- Soberanía alimentaria

- Territorio

\section{Resumo}

Este trabalho tem como objetivo fazer uma contribuição e metodológica para as experiências da relação construída entre contribuição pesquisa, ensino e extensão em uma determinada prática: Programa de Auto Alimentação do Facultad de Ciencias Agrarias-Universidad Nacional de Mar del Plata/Instituto Nacional de Tecnología Agropecuaria-Balcarce.

Esta atividade demonstra o viés da divisão entre extensão, ensino e pesquisa. A dinâmica deste tipo de ação e da comunidade gera ambos os estudantes, profissionais, professores - pesquisadores e produtores assumir papéis alternadamente relevantes em termos de gestão, extensão, pesquisa e ensino, de uma forma não-tradicional, mas necessário para a realização e sustentabilidade dos projectos acordados, levantando questões que fazem a formação praxiológico, não apenas uma disciplina, mas envolveciência holística como um ser histórico processo.

Palavras-chave

- Extension

- Pesquisa

- Ensino

- Soberania alimentar

- Território

\section{Para citación de este artículo}

Verón, J. Hamdan, V. y Natizon, P. (2016). El programa

Autoproducción de Alimentos. Un espacio de intersección entre extensión, investigación y docencia. En Revista +E versión digital, (6), pp. 240-247. Santa Fe, Argentina: Ediciones UNL. 


\section{Introducción}

El presente trabajo pretende realizar una contribución teóricometodológica a las prácticas de extensión a partir de la relación que se construye entre investigación, la docencia y la extensión en una experiencia concreta: Programa Autoproducción de Alimentos (PAA) de la Facultad de Ciencias Agrarias (FCA) de la Universidad Nacional de Mar del Plata (UNMdP) y del Instituto Nacional de Tecnología Agropecuaria-Balcarce (INTA-Balcarce).

El PAA es un programa de extensión de la FCA-UNMdP en articulación con el INTA-Balcarce que surge a partir de la reflexión de un grupo de estudiantes y docentes-investigadores sobre el rol que la Universidad debía cumplir durante la crisis de 2001. En este sentido, partimos de reconocer la organización comunitaria que se estaba construyendo en la sociedad a raíz de la crisis antes mencionada y se propuso como objetivo acompañar estos procesos y brindar herramientas, en este caso desde la Agricultura Urbana y Periurbana (AUP), para contribuir y fortalecer la autonomía y la organización comunitaria de vastos sectores en situación de vulnerabilidad de las ciudades de Mar del Plata y Balcarce (provincia de Buenos Aires), no solo en cuanto a cubrir una parte de las necesidades de alimentos básicos de la familia sino también como un instrumento para la generación de ingresos.

La idea de generar propuestas que superasen el asistencialismo y de trabajar con estos sectores, y no para ellos permite una construcción colectiva y la apropiación de todos los actores del proyecto, convirtiéndonos todos en sujetos de transformación de la realidad emergente.

En lo que respecta al PAA como dispositivo, lo innovador y a la vez complejo desde un comienzo fue la participación activa de los estudiantes y profesionales con los docentes-investigadores. Esta actividad fue la impulsora de proyectos de investigación específicos, o sea, a partir de las demandas del territorio. Las múltiples dimensiones, tanto socioeconómicas como productivas, son fundamento del abordaje interdisciplinario con la clara intención de aportar a la comprensión mutua de las problemáticas y construir acciones colectivas juntamente con los protagonistas tendientes a su resolución. De esta manera, la extensión, la docencia y la investigación se enriquecen mutuamente y no solamente mejoran la calidad del ámbito universitario sino que también aportan a la construcción de una Universidad comprometida con las problemáticas de la sociedad en la que está inmersa.

\section{Orígenes del Programa Autoproducción de Alimentos}

El capitalismo ha experimentado una crisis económica mundial a partir de la década del '70. La respuesta a esta crisis consistió, a lo largo de los ' 80 y '90, en una sucesión de ataques contra el trabajo y las instituciones que lo apoyan:

"un amplio movimiento de quiebras y suspensiones de pagos, una vertiginosa espiral de concentración y centralización, y una urgente compulsión a encontrar nuevos mercados y nuevas fuentes de recursos para el imparable poder de los capitales mundiales dominantes. La política económica neoliberal surgió de la necesidad de apoyar y coordinar estas respuestas típicas de la clase capitalista". (Shaikh, 2001:1)

Para el caso de Argentina, en el año 2001 se evidencia, a partir de la crisis político-institucional y del descontento social, el agotamiento de un modelo socioeconómico que genera pobreza y exclusión sin precedentes. Desde entonces, si bien se reconoce que hay un crecimiento económico entre 2003 y 2008, luego se produce una desaceleración del mismo, y que los índices de pobreza, desocupación y subocupación han disminuido, éstos continúan siendo altos. La Argentina convive en la paradoja de ser reconocida como proveedor de alimentos y simultáneamente mostrar parte de su población con pobreza, indigencia, desnutrición. En este marco, las huertas urbanas para autoconsumo, que se vienen desarrollando en ciudades de todo mundo desde hace siglos, pasan a ser una estrategia de sobrevivencia para vastos sectores de la población tanto a nivel individual-familiar como comunitario, como fue el caso de las ciudades de Mar del Plata y Balcarce (Verón, 2009). Sin embargo, los procesos de participación que se generan se vuelven una vía alternativa no solo de sobrevivencia sino de construcción de otras formas socioeconómicas sustentables (Coraggio, 2004). En este sentido, "si la cuestión social es desigualdad, es también rebeldía, porque implica a los sujetos que a ella se resisten y a ella se oponen" (lamamoto, 2002:33). En este marco, podemos hablar de los Nuevos Movimientos Sociales, los movimientos de desocupados, fábricas recuperadas, organizaciones campesinas e indígenas, asambleas multisectoriales en defensa del medioambiente, organizaciones de derechos humanos, etc. Así, cobra relevancia el estudio de estas formas de acción colectiva, reconociendo que, con un fuerte un anclaje territorial, las mismas recrean formas nuevas de organización, nuevas formas de acción no convencionales.

Tal como se planteó anteriormente, el PAA en las ciudades de Mar del Plata y Balcarce nació a partir de reflexiones acerca del rol que podía jugar la Universidad como parte de la comunidad en la situación de crisis que se vivía en el año 2001. Se manifestó el interés de acompañar los procesos de organización que se habían iniciado en las comunidades, aportando herramientas para contribuir y fortalecer la autogestión y la organización comunitaria de vastos sectores en situación de alta vulnerabilidad. Surgió de un grupo 
66

PAA se cuestiona la concepción predominante en el Sistema Científico Técnico y Educativo Argentino, el de la "neutralidad" de la ciencia y del conocimiento.

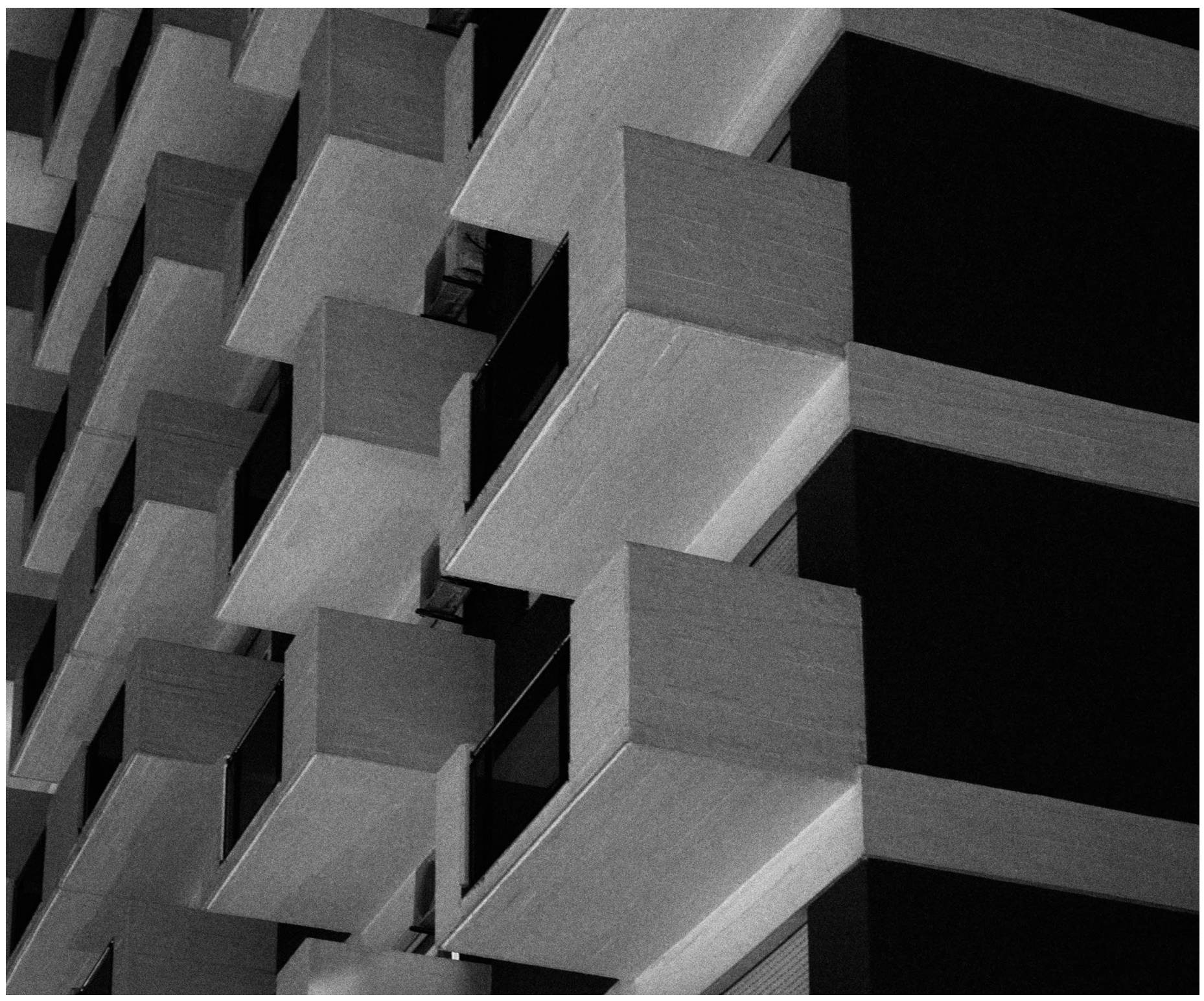

() Oscar Dechiara 
conformado por estudiantes avanzados de Ingeniería Agronómica (sumándose con el tiempo estudiantes de otras carreras) y por un equipo interdisciplinario de docentes-investigadores de la Unidad Integrada Balcarce ${ }^{1}$ involucrados en un proyecto de investigación que decidieron trabajar junto a las comunidades en situación de pobreza de distintos barrios de las ciudades de Mar del Plata y Balcarce.

Es importante resaltar que el PAA se originó en plena crisis 2001-2002, momento en que la ciudad de Mar del Plata lideraba las cifras de la desocupación en Argentina con el 24,1 \%, contra un desempleo global, en el país, que rondaba el 15 \% (INDEC, 2002). Actualmente, según el INDEC (2016), Mar del Plata es la única ciudad del país cuya tasa de desocupación es de dos dígitos y es una de las ciudades en la que más se deterioró el empleo en los últimos tiempos, con de un 11,8\% para el último trimestre de 2015. En este marco, el PAA surgió con el objetivo de generar propuestas superadoras a la realidad emergente de los sectores más vulnerables. El grupo de trabajo decidió poner al servicio de la comunidad los conocimientos y potencialidades de la Universidad y fomentar, mediante la articulación, el accionar de otros organismos de desarrollo, como el INTA. EI PAA apuntó a promover el desarrollo de estrategias que, a partir de la agricultura urbana, no solo permitiera cubrir una parte de las necesidades de alimentos básicos de las familias sino también ser un instrumento para la generación de ingresos y de organización comunitaria.

Se pensó que la agricultura urbana podía constituirse en un medio para el fortalecimiento de las redes sociales e institucionales que a su vez facilitara el acceso a mayor información y a servicios que permitan revertir las situaciones de exclusión. La idea central fue: el Programa cumplirá una función significativa solo si es capaz de superar el asistencialismo y es apropiado por los actores, particularmente por los nuevos movimientos sociales emergentes, como herramienta para consolidarse como actores sociales, con aumento de autonomía, capacidad de resistencia y de demanda de una nueva sociedad.

Se pretende que sectores en situación de pobreza y excluidos del circuito formal de trabajo logren una mejor integración a la sociedad mediante el desarrollo y consolidación de procesos autogestivos y solidarios, constituyéndose así en actores protagónicos de su propio desarrollo. Mediante la generación de espacios participativos y de organización comunitaria y con el fin de complementar el ingreso familiar y la inclusión social, se promueve: la autoproducción de alimentos agroecológicos y su consumo; la producción de excedentes para venta; el fortalecimiento de redes alternativas de comercialización y apropiadas a la agricultura agroecológica y la medicina natural y el autocuidado de la salud.

Consideramos importante destacar que al interior PAA se cuestiona la concepción predominante en el Sistema Científico Técnico y Educativo Argentino, el de la "neutralidad" de la ciencia y del conocimiento. En primer lugar, por la acción de aportar y desarrollar tecnología directamente orientada a sectores desfavorecidos de la sociedad. La investigación agronómica está guiada, prácticamente en su totalidad, por objetivos óptimos de productividad que no contemplan su adaptabilidad a las condiciones de los sectores sociales desfavorecidos, los pequeños productores rurales y la agricultura urbana. El Programa que desarrollamos es un trabajo de extensión que articula con la acción de un grupo interdisciplinario de investigación-acción que trabaja en desarrollo tecnológico, orientado por los seguimientos de huertas y por las demandas que van surgiendo del mismo Programa. Se trata de una innovación significativa en la manera de desarrollar tecnología en nuestras instituciones (Hamdan y Verón, 2007a).

Otra dimensión del cuestionamiento a la neutralidad del conocimiento científico es que tanto el proyecto de investigación como el proyecto de extensión parten de una crítica al modelo de agricultura industrial y se inscriben claramente en una concepción agroecológica de producción (Hamdan y Verón, 2007b).

\section{Equipo de trabajo y modalidad de abordaje con la comunidad} EI PAA inició su trabajo en los barrios a partir de la conformación de un grupo de estudiantes avanzados de la Facultad de Ciencias Agrarias, al cual se sumaron, con el tiempo, estudiantes de otras unidades académicas de la UNMdP (Biología, Geografía, Ciencias de la Salud y Servicio Social, Arquitectura y Diseño, Ingeniería y Ciencias Económicas). Se plantearon dos tipos de participación: Pasantías Educativas Internas (PEI) que cobraban un estipendio mensual para gastos de estudio, y, por otro, estudiantes que trabajaban en forma voluntaria y la labor comunitaria de profesionales de la actividad privada. Los estudiantes y profesionales jóvenes (en la mayoría de los casos estos profesionales han acumulado experiencia en la temática por haber pasado como estudiantes por el PAA) fueron los pilares centrales en la vinculación con la comunidad y el sostenimiento del funcionamiento del Programa.
1) La Unidad Integrada Balcarce está conformada por la FCA de la UNMdP y el INTA-Balcarce. Desde 1962 se desarrollan actividades conjuntas de docencia universitaria, investigación, extensión y capacitación. Funciona como un sistema integrado de forma- ción académica, generación y transferencia de conocimientos y tecnología en beneficio de los estudiantes de grado y de posgrado y de los distintos actores relacionados con el sector agroindustrial y agroalimentario 


\section{6}

la función del equipo técnico (estudiantes

y profesionales) es estimular la participación

comunitaria en la realización de los

emprendimientos de agricultura agroecológica
Bajo la modalidad de extensión participativa se buscó la promoción, capacitación y apoyo a la autoproducción de alimentos y su comercialización desde una perspectiva agroecológica, teniendo en cuenta que la misma no solo involucra aspectos productivos sino también toma como fundamento el componente social de dicha producción y de organización.

La función del equipo técnico (estudiantes y profesionales) es estimular la participación comunitaria en la realización de los emprendimientos de agricultura agroecológica (huertas, granjas y viveros) y son responsables de la promoción, asesoramiento técnico y organizacional de los mismos. El trabajo incluye además el reconocimiento del territorio, de los actores y de las organizaciones que participan en cada lugar. Intercambian conocimientos con los productores, capacitan y asesoran en aspectos teóricos y prácticos, asisten en la planificación y gestión de los emprendimientos en marcha que así lo requieren propiciando el protagonismo y la autoorganización de la comunidad. Por lo tanto, su trabajo no se reduce a las cuestiones técnicas sino que implica realizar un gran esfuerzo en cuanto a formación en dinámica de grupos. Facilitan la vinculación de los actores entre sí y con las instituciones para organizarse, gestionar, capacitar y demandar soluciones.

\section{Las misiones de la universidad: reflexiones sobre la investigación, extensión y docencia}

\subsection{En torno a la investigación y los investigadores}

Partimos de un cuestionamiento claro acerca de la "neutralidad de la ciencia". La concepción predominante en el sistema científico y educativo argentino de la neutralidad de la ciencia y del conocimiento fue cuestionada y abordada críticamente a partir del desarrollo del PAA.

A partir del trabajo que se iniciaba en el Programa, se conviene desde el inicio que una de sus actividades centrales debía ser la promoción del desarrollo de tecnologías apropiadas para un nuevo actor productivo, los productores urbanos, quienes emergen en el marco de una fuerte crisis socio-económica. Un nuevo actor que presentaba ciertas particularidades, no contempladas en el ámbito científico-académico: ser parte del sector más vulnerable de la sociedad desocupados y subocupados urbanos; producir alimentos para autoconsumo y/o venta a partir del desarrollo de la agricultura urbana y ser su producción, en la mayoría de los casos, de base agroecológica (Hamdan y Verón, 2007).

Tanto los proyectos de investigación que articulan con el PAA como el mismo proyecto de extensión parten de una crítica al modelo de agricultura industrial y se inscriben claramente en una concepción agroecológica de producción. Mediante una estrecha vinculación del proyecto de investigación con la actividad de extensión se comienza a incursionar en el desarrollo tecnológico apropiable a partir de la modalidad de investigación-acción, orientado por los seguimientos de proyectos productivos y por las demandas que van surgiendo del mismo Programa.

Esta forma de desarrollar tecnología adoptada por el equipo, que tiene la particularidad de ser una metodología de transformación y aprendizaje mutuo, ha sido puesta en práctica hace pocos años en el ámbito universitario por un reducido número de investigadores debido a que habitualmente es cuestionada, calificándola como carente de rigor científico. Para el equipo de trabajo, tanto el de investigación como el de extensión, es el único medio de lograr una verdadera apropiación por parte de los actores.

Una de las principales limitantes en el ámbito científico, que no favorece a una mayor participación de docentes-investigadores en las actividades del PAA, es enfrentarse a este desafío que implica una forma distinta, y necesaria, de llevar adelante el proceso de investigación. Es una investigación científica aplicada, con una mirada orientada a problemas concretos y sentidos por la sociedad, problema complejo que es necesario abordarlo conjuntamente en forma interdisciplinaria y con los mismos actores como participantes, única vía de contribuir a una sociedad más inclusiva, respondiendo en forma apropiada a una demanda concreta de la comunidad. Implica diversos grados de interacción con los destinatarios de la tecnología, y que deriva en una actividad de intercambio cognitivo, una puesta en común entre saberes populares y científicos.

La articulación que se ha generado entre docentes-investigadores, productores, estudiantes y profesionales del PAA es una luz verde a la solución de problemáticas reales de los territorios. Lo innovador de esta experiencia es la participación activa de los estudiantes 
y profesionales del PAA con los docentes-investigadores de la institución, siendo los primeros quienes demandan las problemáticas a resolver que surgen de la experiencia de terreno y los segundos, quienes gradualmente aceptan esta demanda, incorporando las mismas en sus proyectos de investigación. Esta actividad no solo fue la impulsora de proyectos de investigación específicos en la temática, por lo cual es destacable la enorme producción científica generada en estos 13 años de desarrollo del Programa más de 100 publicaciones en diferentes eventos científicos nacionales e internacionales, sino también de numerosas tesis de grado, de posgrado y estadías de profesionales internacionales en el marco de su formación de grado y posgrado.

\subsection{En torno a la educación: impacto sobre los estudiantes y docentes}

Las características señaladas del Programa lo hacen susceptible de producir repercusiones positivas para la discusión sobre las características de la formación de los estudiantes de la FCA y de otras carreras de la Universidad. De hecho, están descubriendo una realidad y un modo de trabajar que no les es aportado desde las aulas. Esta situación está siendo incorporada como un debate en el seno de nuestra Universidad.

El rol del PAA siempre fue impulsar y facilitar acciones concretas a la solución de las problemáticas. Por esta razón, si bien se pueden definir lineamientos de acción futuros, en ocasiones, tanto en función de las necesidades del momento como de una evaluación de las posibilidades de implementación, las líneas de acción se modifican y se readecuan a las posibilidades concretas del momento. Esta modalidad de trabajo con la comunidad introduce un cambio muy importante en la formación de los estudiantes, ellos participan de un modo de trabajo no impartido por la propia Universidad -salvo excepciones.

Son destacables la fuerte articulación que se ha logrado entre los integrantes del PAA con la comunidad y el vínculo muy especial que se ha construido. Los mismos productores, cuando se refieren al PAA, se refieren a una gran familia, a que "estamos juntos en las buenas y en las malas".

Pero si bien la Universidad trata de responder a nuestras demandas como actividad universitaria, creemos que falta un largo camino a recorrer para lograr la institucionalización que es necesaria para su continuidad.

Respecto del impacto sobre los estudiantes y profesionales, han desarrollado capacidades múltiples y de difícil adquisición en las aulas con la formación tradicional. El trabajo del PAA implica un aprendizaje constante. A partir de reflexiones realizadas por estudiantes que pasaron por el PAA se visualiza que el mismo no solo posibilita una experiencia laboral sino que va mucho más allá: se vislumbran otros horizontes profesionales (excediendo la disciplina de la carrera que cursan); un aprendizaje que sus carreras no contienen, el de las relaciones humanas, el del esfuerzo conjunto y solidario, el de la confianza, el de las experiencias de vida de la gente, el trabajo en equipo interdisciplinario, la construcción colectiva del conocimiento y el descubrimiento de una habilidad vocacional desconocida, enmarcada o no en sus respectivas carreras. Sin embargo, por la voluntad, constancia y pasión de los integrantes del Programa y de algunos docentes-investigadores, se han cristalizado reconocimientos aislados en diferentes carreras a través de trabajos concretos como: tesis de grado, la figura de pasantía con créditos, becas de extensión rentadas, tesis de posgrado, trabajos prácticos y/o finales de algunas asignaturas, presentación de trabajos a congresos y jornadas o, simplemente, ganas de contar lo que se realiza dentro del PAA en cada ámbito académico en donde se encuentra.

Lo anterior posibilitó la inserción de temáticas importantes como la agroecología, la agricultura urbana, el desarrollo territorial sustentable, el compromiso social, trabajo participativo y solidario, entre otras; conceptos que, sin estar aún instalados en algunas asignaturas, estudiantes que han pasado o todavía permanecen en el PAA introducen el debate en las aulas con total convencimiento frente a docentes y a sus propios pares.

Por otro lado, el tránsito de los estudiantes, ya sea como voluntarios, como pasantes rentados o becarios de extensión, hace referencia a aprender a construir con el otro. Se trata de aprendizajes que las aulas no pueden brindar, que es posible a partir un vínculo de confianza, una relación con esos otros, que los libros pueden describir y comprender pero no enseñan a cómo construirlo y que solo es posible, a nuestro entender, por la experiencia directa, mediante esa relación cara a cara, mate de por medio, abrazo por 
los logros o por los fracasos, compartiendo espacios en los cuales todos tienen algo para aportar y también aprender. Es un desarrollo y crecimiento no solo profesional sino sobre todo personal. ${ }^{2}$

A los aspectos mencionados, desde un punto de vista de la formación profesional, se suma que el PAA promueve y prioriza la capacitación que puedan adquirir los estudiantes a través de su participación en diferentes eventos relacionados con las temáticas de interés para el PAA y que les resultaría más difícil de hacer desde sus respectivas carreras (congresos científicos, congresos y jornadas de extensión así como la realización de cursos y seminarios), en tanto que en numerosas ocasiones son protagonistas como expositores de trabajos que han realizado en el programa o simplemente exponiendo las actividades que se realizan.

A modo de síntesis, es indiscutible la relevancia que tendría en la formación integral de los estudiantes el reconocimiento curricular de este tipo de prácticas que desarrollan esta perspectiva. La falta de reconocimiento a este tipo de actividades por parte de la institución se convierte en un obstáculo muy importante para la conformación de un grupo comprometido de docentesinvestigadores que trabaje en forma conjunta con los estudiantes. Este aspecto es una de las limitantes que experimenta hoy el PAA, entre otras resultantes de la falta de una genuina institucionalización. No se trata solo de generar los actos administrativos que avalen su existencia, sino de apropiarse del proyecto y generar los espacios e instancias necesarios para su institucionalización, además del aporte de los recursos humanos y económicos que conviertan al proyecto en algo más que la suma de voluntades individuales.

\subsection{Práctica de extensión: impacto sobre la comunidad}

En cuanto al impacto social, la modalidad de abordaje con la comunidad es lo que ha permitido promover: la pertenencia, la autonomía, la solidaridad, la organización comunitaria Además de los logros cuantitativos, en cuanto a cantidad de emprendimientos que hoy acompaña el Programa; el aspecto más relevante a señalar, es que los "productores" son protagonistas de la experiencia, sujetos activos que participan de las acciones a la par del equipo de trabajo del PAA.

Son parte de la puesta en marcha de todas las actividades de promoción comunitaria que se llevan adelante en el marco del PAA: ferias, fiestas y exposiciones barriales, charlas y talleres de capacitación, difusión de la agroecológica, promoción de la salud, actividades culturales y recreativas. El nivel organizativo alcanzado, el grado de compromiso adquirido y el trabajo comunitario, solidario y respetuoso por sus compañeros, entre otros, son los principales aspectos a destacar como capacidades desarrolladas en la comunidad.

La modalidad de abordaje en el medio está basada en una estrategia de fortalecimiento comunitario. Articulación efectiva y constante bajo una modalidad relacional y organizativa promovida desde lo sociocomunitario. Estudiantes, profesionales y docentesinvestigadores reunidos interdisciplinariamente en torno a la misma problemática; estudiantes y docentes-investigadores de diferentes carreras reunidos con los productores, con la participación activa y protagónica de organizaciones sociales barriales, ONG, organismos públicos, municipalidades, salas de salud, médicos y otros especialistas de la actividad privada y empresas privadas solidarias de la ciudad. Es una acción conjunta comunitaria no solo participante sino más bien protagónica.

\section{A modo de síntesis: espacios comunes entre la extensión, la investigación y la docencia}

La actividad del Programa constituye en sí una evidencia de la parcialidad que conlleva la fragmentación entre las tres misiones de la universidad normalmente reconocidas. El espacio de intersección que se visualiza en el Gráfico 1 simboliza los espacios comunes entre las tres misiones de la universidad y la sociedad, espacios de construcción colectiva, de esfuerzos y logros. La dinámica de
2) A lo largo de estos 13 años de trabajo ininterrumpido se han formado en el PAA a través de pasantías rentadas y becas de Extensión: 38 estudiantes de la Facultad de Ciencias Agrarias, 6 estudiantes de la Facultad de Ciencias Exactas y Naturales, 2 de la Facultad
Ciencias de la Salud, 3 de la Facultad de Ciencias Económicas y Sociales y 2 de la Facultad de Humanidades. Además, han participado desde la cátedra de Unidades Prácticas de la carrera de Licenciatura en Servicio Social 15 estudiantes de $2^{\circ}, 3^{\circ}$ y $4^{\circ}$ años. 
este tipo de acciones en y con la comunidad genera que tanto estudiantes, profesionales, docentes-investigadores y productores se asuman indistintamente en roles relevantes en cuanto a gestión, extensión, investigación y docencia, en una forma no tradicional pero necesaria para la concreción y sustentabilidad de los proyectos consensuados, planteando interrogantes que hacen a la formación praxiológica no solo de una disciplina sino de la ciencia de manera integral como proceso histórico "que está siendo".

\section{Gráfico 1. Espacio de intersección}

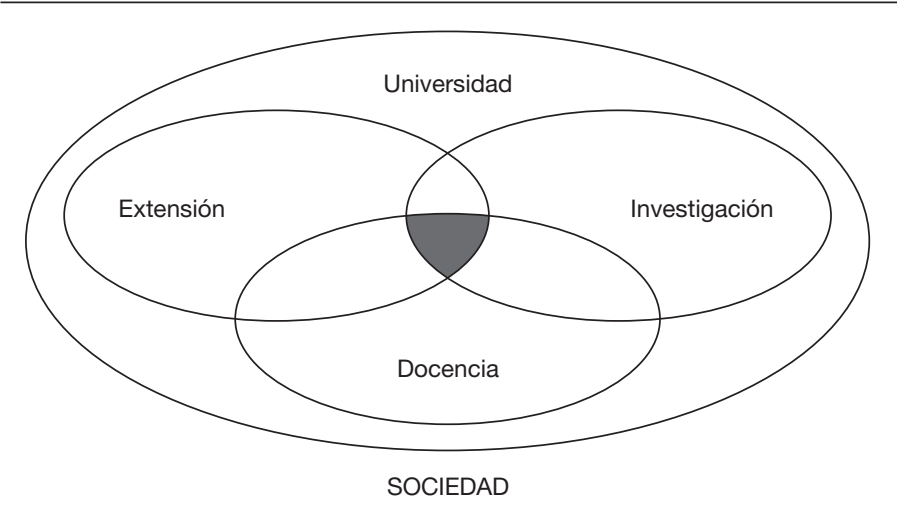

Fuente: elaboración propia

La posibilidad de acompañar y aportar desde la universidad pública con herramientas que contribuyan a expresar la capacidad de autonomía y el protagonismo de los sectores sociales vulnerados da una especial perspectiva a la extensión, a la investigación y a la docencia y no solamente mejora la calidad académico-científica del ámbito universitario sino que aporta a la construcción de una universidad comprometida con las problemáticas de la sociedad en la que está inmersa.

\section{Referencias bibliográficas}

Coraggio J. L. (2004). De la Emergencia a la Estrategia. Buenos Aires: Espacio. lamamoto, M. (2002). Fenómeno social de la exclusión. En AA. VV. Trabajo Social y Mundialización. Buenos Aires: Espacio.

Hamdan, V. y Verón, J. (2007a). El Programa de Autoproducción de Alimentos de la UNMdP - Reflexión en torno al impacto en las misiones institucionales de la Universidad Educación, Investigación y Extensión. Jornada Internacional Alemania, Kenia y Argentina: "Agricultura urbana y Universidad", 10 de octubre, Facultad de Agronomía de la Universidad de Buenos Aires.

Hamdan, V. y Verón, J. (2007b). Reflexiones a partir de un proceso de Aprendizaje-Servicio. El caso del Programa de Autoproducción de Alimentos de la UNMdP. $10^{\circ}$ Seminario Internacional de Aprendizaje y Servicio Solidario. Facultad de Derecho UBA. Buenos Aires, 30 y 31 de agosto.

INDEC (2002). Índice de desocupación de Aglomerado Mar del Plata-Batan. Argentina.

INDEC (2016). Índice de desocupación de Aglomerado Mar del Plata-Batan. Argentina.

Shikh, A. (2001). La explicación de la inflación y el desempleo: una alternativa a la teoría económica neoliberal. En Guerrero, D. (Ed.), Macroeconomía y crisis mundial. Madrid: Trotta.

Verón, J. (2009). Indicadores de ciudadanía. Un aporte metodológico para evaluar la sustentabilidad de procesos participativos generados en el marco del Programa de Autoproducción de Alimentos. El caso de la Feria Verde de la ciudad de Mar del Plata. Tesis de grado. Facultad de Ciencias de la Salud y Servicio Social, Universidad Nacional de Mar del Plata. 